\title{
Thioredoxin-interacting protein: an oxidative stress-related gene is upregulated by glucose in human prostate carcinoma cells
}

\section{See-Tong Pang, Wen-Chi Hsieh', Cheng-Keng Chuang, Chun-Hsiang Chao', Wen-Hui Weng ${ }^{2}$ and Horng-Heng Juang ${ }^{1}$}

Division of Urology, Department of Surgery, Chang Gung Memory Hospital, Kwei-Shan, Tao-Yuan 333, Taiwan, ROC

'Department of Anatomy, Chang Gung University, 259 Wen-Hua 1st Road, Kwei-Shan, Tao-Yuan 333, Taiwan, ROC

${ }^{2}$ Department of Chemical Engineering \& Biotechnology, Institute of Biotechnology, National Taipei University of Technology, Taipei, Taiwan, ROC

(Correspondence should be addressed to H-H Juang; Email: hhj143@mail.cgu.edu.tw)

\begin{abstract}
Thioredoxin-interacting protein (TXNIP), also known as vitamin- $\mathrm{D}_{3}$ upregulated protein 1 , interacts with reduced thioredoxin. This protein modulates the cellular redox state and plays a role in stress-induced cellular apoptosis. This study examined TXNIP gene expression in prostate cancer cells. In vitro studies by immunoblot assay have shown that elevated glucose levels (1-15 mM) upregulate TXNIP gene expression two- to fourfold in human prostate carcinoma cells (LNCaP) and hepatocellular carcinoma cells (HepG2). Transient gene expression assays reveal that the promoter activity of the TXNIPgene is upregulated by glucose, 3-O-methylglucose, and maltose, but not by mannitol. These results suggest that glucose and 3-O-methylglucose induce TXNIP expression through both glucose metabolism-dependent and -independent pathways. Cotransfection of a plasmid expression carbohydrate response element-binding protein (ChREBP) with a TXNIP reporter vector into LNCaP cells dramatically enhances reporter activity in a low glucose (1 mM) condition. The effects of glucose are apparently mediated in a region located -341 to $-324 \mathrm{bp}$ upstream of the translational starting point of the TXNIP gene as indicated by $5^{\prime}$-deletion and site-directed mutagenesis reporter assays. Mutation of the putative carbohydrate response element (ChoRE) from CACGAGGGCAGCACGAG to TTTGAGGGCAGCACGAG abolishes glucose upregulation of TXNIP promoter activity. The present study demonstrates that TXNIP is transcription induced in both LNCaP and HepG2 cells in an increased glucose metabolism-dependent or -independent response, and a putative glucose regulatory system including ChREBP and ChoRE is needed for glucose-induced TXNIP gene in human prostate carcinoma cells.
\end{abstract}

Journal of Molecular Endocrinology (2009) 42, 205-214

\section{Introduction}

Thioredoxin-interacting protein (TXNIP), also known as vitamin- $\mathrm{D}_{3}$ upregulated protein 1 (VDUP1) or thioredoxin-binding protein-2 was first isolated from HL-60 cells following 1,25-dihydroxyvitamin $\mathrm{D}_{3}$ treatment (Chen \& DeLuca 1994). TXNIP is known to negatively regulate thioredoxin (TRX) that further regulates mammalian signal transduction (Gulati et al. 2001, Nishiyama et al. 2001). Therefore, recent studies have gradually clarified the involvement of TXNIP in important cellular events such as vascular inflammation and proliferation (Schulze et al. 2002, Yamawaki et al. 2005), collagen expression in mesangial cells (Kobayashi et al. 2003), melanoma metastasis (Goldberg et al. 2003), mucosal immunity in gastrointestinal cancer (Ikarashi et al. 2002), osteoclast differentiation (Aitken et al. 2004), cardiac hypertrophy, and myocardial ischemia (Wang et al. 2002, Yoshioka et al. 2004, Xiang et al. 2005), decidualization of endometrium (Simmons \& Kennedy 2004), renal carcinogenesis (Dutta et al. 2005), T-cell leukemia leukemogenesis and immunology (Nishinaka et al. 2004, Lee et al. 2005), lung growth (Filby et al. 2006), cell proliferation, and the aging process (Yoshida et al. 2005, Wang et al. 2006). Since TXNIP expression is often suppressed in tumor cells, this protein apparently has tumor-suppressive properties. TXNIP is a novel factor in p27 $27^{\text {kip } 1}$ stability via regulation of JAB1. Transfection of TXNIP induces cell cycle arrest at the G0/G1 phase in several types of tumor cells (Han et al. 2003, Jeon et al. 2005). However, TXNIP antisensetransfected murine melanoma cells exhibit decreased levels of intracellular reactive oxygen species and inhibited cell proliferation (Song et al. 2003). TXNIP function and gene regulation in human prostate cells require further clarification. Early studies have indicated 
that TXNIP expression is negatively regulated by androgens in murine prostate cells (Pang et al. 2002).

Recent studies have indicated that TXNIP is important in metabolic responses to feeding. Oligonucleotide microarray analysis from two independent laboratories indicated that TXNIP is a glucose-upregulating gene in human pancreatic islets and rat-1 fibroblasts (Hirota et al. 2002, Shalev et al. 2002). Analyses of HcB-19 mice or TXNIP knockout mice have also revealed the critical role of TXNIP in the integrated regulation of glucose and lipid metabolism during fasting (Hui et al. 2004, Sheth et al. 2005, Oka et al. 2006). Furthermore, a recent study of genome-wild expression profiles has indicated that the human TXNIP gene is important in regulating peripheral glucose metabolism (Parikh et al. 2007). A study using human pancreatic $\beta$-cells further revealed glucose stimulation of TXNIP expression. Transient gene expression indirectly suggests that glucose induces TXNIP expression through a putative carbohydrate response element (ChoRE) that does not contain the consensus sequence of ChoRE in the promoter of the TXNIP gene (Minn et al. 2005). Interestingly, this study indicated that upregulation of the TXNIP gene by glucose does not require glucose metabolism.

This study characterizes TXNIP expression in human prostate carcinoma cells and the regulatory mechanism of glucose on TXNIP expression.

\section{Materials and methods}

\section{Cell cultures and chemicals}

Prostate cancer cell lines PZ-HPV-7, CA-HPV-10, LNCaP, PC-3, DU145, and hepatocarcinoma cells (HepG2) were obtained from the American type culture collection and maintained as described previously (Feng et al. 2005, Tsui et al. 2008). PZ-HPV-7 cells were derived from epithelial cells cultured from normal tissue from the peripheral zone of the prostate. CA-HPV-10 cells were derived from prostatic adenocarcinoma cells of Gleason grade 4/4. The cells were immortalized by transformed human papillomavirus (Weijerman et al. 1994). Glucose, maltose, mannitol, and 3-O-methylglucose were purchased from Sigma. FCS was purchased from HyClone (Logan, UT, USA). All culture media and reagents were purchased from Life Technologies. Cells were cultured in RPMI 1640 containing $10 \%$ FCS, and the medium was replaced twice a week. In the following experimental studies, cells were cultured in RPMI1640 glucose-free medium with $10 \%$ FCS for $4-6 \mathrm{~h}$ to glucose starvation and then cultured in the same medium with various concentrations of glucose for 16-24 h.

\section{Semi-quantitative reverse transcription-PCR}

Total RNA was isolated with Trizol reagent; cDNA was synthesized by the superscript III preamplification system, following the manufacturer's instructions (LifeTechnologies). Excess RNA was degraded by RNase $\mathrm{H}$ treatment. TXNIP-P and TXNIP-R primers (Table 1) were used to amplify sequences specific to human TXNIP mRNA (GenBank accession no. NM_012391). The quality of cDNA was verified by controlled reactions using primers derived from $\beta$-actin-P and $\beta$-actin-R (Table 1). PCR was performed in a thermal cycler (Thermolyne, Dubuzue, IA, USA) under the following parameters: 30 cycles at $94^{\circ} \mathrm{C}$ for $0.5 \mathrm{~min}, 55^{\circ} \mathrm{C}$ for $1 \mathrm{~min}$ and $72^{\circ} \mathrm{C}$ for $1 \mathrm{~min}$. PCR products, $615 \mathrm{bp}$ of TXNIP cDNA fragments and $720 \mathrm{bp}$ of $\beta$-actin cDNA fragments, were separated by $2 \%$ agarose gel electrophoresis and visualized by ethidium bromide staining.

\section{Expression vector constructs}

The c-Myc (MGC:5184) and ChoRE-binding protein (ChREBP; MGC:9556) cDNA vectors were purchased from the Human Genome Mapping Project Resource Centre, UK. Human c-Myc cDNA was linearized by cutting with Kpn I and Xho $I$ and ligation with the overexpression vector pcDNA3 (Invitrogen), resulting in insertion of c-Myc cDNA in the polyadenylate region controlled by the CMV promoter (pcDNA3-cMyc). Purchased ChREBP cDNA (MGC:9556) vector does not contain full-length ChREBP cDNA but it does have $279 \mathrm{bp}$ deletion (exon 5 and exon 6). Therefore, the full-length human ChREBP overexpression vector was constructed by digested ChREBP cDNA vector with Eco $R V$ plus Xmn I and Bas $H I$ plus Xho I, which led to two cDNA fragments, 344 and $2645 \mathrm{bp}$. Another $553 \mathrm{bp}$ cDNA fragment was synthesized using RT-PCR from HepG2 cells by two primers ChREBP-P and ChREBP-R (Table 1) and digested with Xmn I and Bas HI. These three cDNA fragments were ligated and cloned into the pcDNA3 overexpression vector after digestion with $E c o R V$ and $X$ ho I. Proper ligation was confirmed by extensive restriction mapping and sequencing.

Table 1 Primers used in this study for RT-PCR

\section{Sequence}

Name

TXNIP-P

TXNIP-R

$\beta$-actin-P

$\beta$-actin- $R$

ChREBP-P

ChREBP-R
5'-AGTTTCCTGCATGTTCATTCCT-3' 5'-GAACTTGAACTCAGGGGCATAC-3' 5'-GAAGATCAAGATCATTGCTCCTCC-3' 5'-GAAGATCAAGATCATTGCTCCTCC-3' 5'-CCAAGTGGAAGAATTTCAAAGG-3 5'-CGGGAGTTGGTAAAGAAATCTG-3' 


\section{Immunoblot assay of human TXNIP and $\beta$-actin}

Cells were treated with different glucose concentrations as indicated. Cells were lysed with lysing buffer $(62.5 \mathrm{mM}$ Tris ( $\mathrm{pH} 6.8), 2 \%$ SDS, $10 \%$ glycerol, $5 \% \beta$-mercaptoethanol and $7 \mathrm{M}$ urea), and protein concentrations of aliquot samples were measured by bicinchoninic acid protein assay (Pierce Biotechnology, Rockford, IL, USA). Equal amounts of protein were loaded onto a $12.5 \%$ SDS-polyacrylamide gel and assayed by ECL detection per manufacturer instructions (Amersham Biosciences). The blot membrane was probed with 1:1000 diluted monoclonal TXNIP antiserum (JY2; MBL International Corp., Woburn, MA, USA) or 1:1000 diluted anti $\beta$-actin antiserum (C11, Santa Cruz Biotechnology, Santa Cruz, CA, USA).

\section{Reporter vector constructs and site-directed mutagenesis}

The reporter vector of the TXNIPgene $(-3104$ to -1$)$ was provided by Dr I. Choi, Korea Research Institute of Bioscience and Biotechnology, Republic of Korea (Han et al. 2003). The reporter vector containing the $5^{\prime}$-flanking region of the human TXNIP gene was cloned by PCR using the GL-2 primer and TXNIP-specific primers (Table 2). Reporter vectors, pChoREm1, and pChoREm2, containing the mutant putative ChoRE were established using a QuikChange site-directed mutagenesis kit (Stratagene, La Jolla, CA, USA). The double-stranded primers used for in vitro site directed mutagenesis were 5'-GGAGCACACCGTCCCCACGCGCCACAGCGATC-3' and $5^{\prime}$-CAATGGGAGGGATGTGTTTGAGGGCAGCACGAGCC-3' respectively (mutation sites are underlined).

\section{Transient transfections and reporter assay}

The LNCaP or HepG2 cells were plated onto 24-well plates at $1 \times 10^{4}$ cells/well one day prior to transfection.

Table 2 Primers used in this study for construction of reporter vectors

\section{Sequence}

$\begin{array}{lll}\text { Name } & & \\ \text { TXNIP-2 } & \text { 5'-ACGCGTTACACTTTCATACC- }^{\prime} \text { CATC-3' }^{\prime} & -1614 \\ \text { TXNIP-3 } & \text { 5'-ACGCGTACTCAGCTCCTAAAG- }^{\prime} & -1102 \\ & \text { CATC-3' }^{\prime} & -921 \\ \text { TXNIP-4 } & \text { 5'-ACGCGTAGAAATTGAAGAGAT- }^{\prime} & -612 \\ & \text { GACAGG-3' }^{\prime} & -624 \\ \text { TXNIP-5 } & \text { 5'-ACGCGTCCA- }^{\prime} & -520 \\ \text { TXNIP-5-1 } & \text { CACCCGCCCTCCGATG-3' }^{\prime} \text {-ACGCGTCAA- } & -420 \\ & \text { CAGCCCCCTCCTTCCCC-3' } & \\ \text { TXNIP-5-2 } & \text { 5'-ACGCGTGTCCACGCGCCA- }^{\prime} & \\ & \text { CAGCGA-3' }^{\prime} & \\ & & \end{array}$

Cells were transiently transfected using the TransFast transfection reagent $(0 \cdot 6 \mu \mathrm{g} /$ well; Promega Biosciences $)$ with $1 \mu \mathrm{g} /$ well of reporter vector and $0.5 \mu \mathrm{g} /$ well of the pCMVSPORT $\beta$ Gal (Life Technologies) for $4 \mathrm{~h}$. Cells were then incubated in RPMI 1640 medium with 10\% FCS overnight. Transfected cells were treated with RPMI 1640 glucose-free medium with $10 \% \mathrm{FCS}$ for $4 \mathrm{~h}$, and then with the same medium at varying concentrations of glucose, as indicated, for an additional $16 \mathrm{~h}$. Cell extracts were assayed for luciferase and $\beta$-galactosidase ( $\beta$-GAL) activity according to the manufacturer's instructions (Promega Biosciences). Luciferase activity was determined by LumiCount (Packard Bioscience, Meriden, CT, USA) and adjusted accordingly for $\beta$-GAL enzymatic activity, as previously described (Juang et al. 2004). However, preliminary studies indicated that cotransfection with high concentrations of ChREBP or c-Myc affects the activity of the $\beta$-galactosidase expression vector (pCMVSPORTßgal; Life Technologies) in transient gene expression assay. Therefore, luciferase activity in these experiments was adjusted using protein concentrations in the whole cell extract, which were measured by bicinchoninic acid protein assay (Pierce Biotechnology).

\section{Electrophoretic mobility shift assay}

An electrophoretic mobility-shift assay (EMSA) was performed as described previously (Tsui et al. 2006). The double-stranded DNA fragment containing the putative ChoRE (5'-TGTGCACGAGGGCAGCACGAGCCTC-3' $)$, was $5^{\prime}$-end labeled with $\gamma$-P $\mathrm{P}^{32} \mathrm{ATP}$ using T4 polynucleotide kinase. Nuclear extracts of LNCaP cells, after treatment with different glucose concentrations, were obtained using NE-PER nuclear and cytoplasmic extraction reagents, per manufacturer instructions (Pierce, Rockford, IL, USA). The $5^{\prime}$-end-labeled ChoRE (ChoRE probe; $5 \mathrm{nM}$ ) was incubated with $2 \mu \mathrm{g}$ nuclear extract (NE) from LNCaP cells in $20 \mu$ binding buffer (25 mM HEPES buffer ( $\mathrm{pH}$ 7.9), $50 \mathrm{mM}$ KCL, 0.05 mM EDTA, 0.5 mM dithiothreitol, $0.5 \mathrm{mM}$ phenylmethylsulfonyl fluoride, and $10 \%$ glycerol) containing $0.5 \mu \mathrm{g}$ poly $(\mathrm{dI}-\mathrm{dC})$-poly $(\mathrm{dI}-\mathrm{dC})$. The binding shift was challenged with 50-fold double-stranded ChoRE or double-stranded mutational ChoREm (5'-TGTGTTTGAGGGCAGCACGAGCC- $3^{\prime}$ ), without labeling the $5^{\prime}$-end with $\gamma$-P ${ }^{32}$ ATP. Protein-DNA complex formations were analyzed on $4 \%$ polyacrylamide gels by autoradiography.

\section{Statistical analysis}

Results were expressed as the mean \pm s.E.M. for at least three independent replications of each experiment. Statistical significance was determined by paired $t$-test analysis and one-way ANOVA using SigmaStat software for Windows (version 2.03; SPSS Inc., Chicago, IL, USA). 

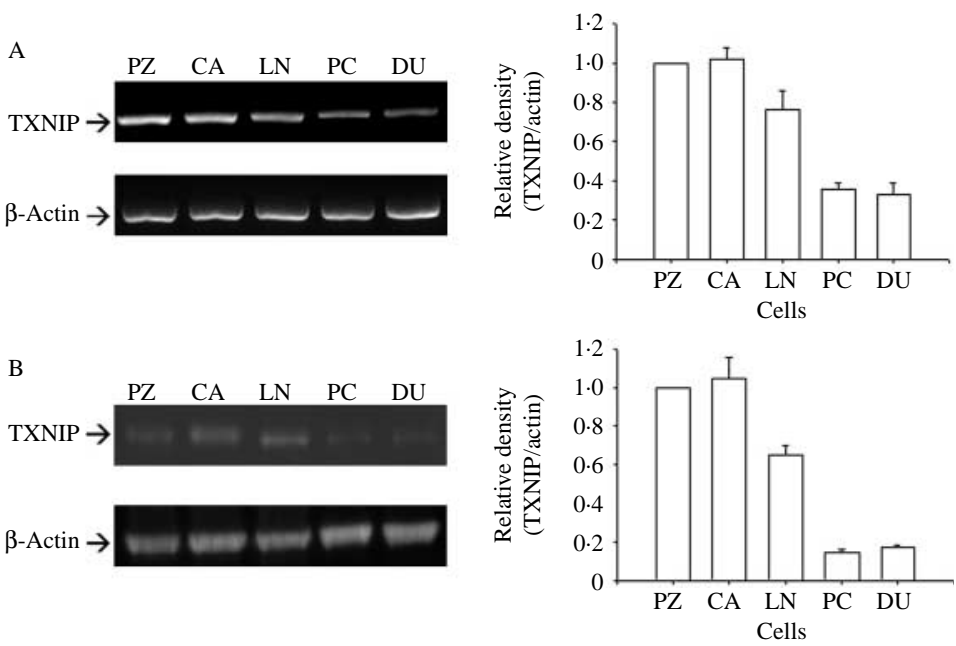

Figure 1 Gene expression of TXNIP in various prostate carcinoma cells. The TXNIP gene expression in different cell lines was determined by (A) RT-PCR and (B) immunoblot assay. PZ, PZ-HPV-7; CA, CA-HPV-10; LN, LNCaP; PC, PC-3; and DU, DU145. Quantitative analysis was done by determining the intensity of each band for TXNIP and $\beta$-actin from three independent experiments. Band intensity was analyzed by the GeneTools program of ChemiGenius (Syngene, Cambridge, UK). Data are presented as the mean density of TXNIP/ $\beta$-actin ( \pm S.E.M.) relative to the mean density associated with the PZ-HPV-7 cells.

\section{Results}

We screened TXNIP expression in five prostate cell lines (PZ-HPV-7, CA-HPV-10, LNCaP, PC-3, and DU145). To synchronize the cell cycle, all prostate cells used in this study were incubated in RPMI media without serum for $24 \mathrm{~h}$ and incubated with RPMI media containing 10\% FCS for another $24 \mathrm{~h}$. RT-PCR (Fig. 1A) and immunoblot assays (Fig. 1B) revealed a high rate of metastasized prostate carcinoma cells (PC-3 and DU145), thus indicating a lower amount of TXNIP gene expression, when compared with non-metastatic (PZHPV-7 and CA-HPV-10) or low-metastatic prostate cells (LNCaP). Transient gene expression and immunoblot assays revealed that elevated glucose (1-15 mM) induced a two- to fourfold upregulation in TXNIP gene expression in human prostate carcinoma cells, LNCaP (Fig. 2A and D). When a plasmid expression ChREBP was cotransfected with the TXNIP reporter vector into LNCaP cells, promoter activity was dramatically $(P<0 \cdot 001)$ enhanced in a low glucose $(1 \mathrm{mM})$ condition. However, overexpression of $\mathrm{c}-\mathrm{Myc}$ did not significantly $(P=0.076)$ induce TXNIP promoter activity in the low glucose $(1 \mathrm{mM})$ condition (Fig. 2B and $\mathrm{C}$ ). Glucose-upregulated TXNIP gene expression was observed not only in prostate cells but also in the HepG2 cells (Fig. 3A and D). Promoter activity was significantly $(P<0 \cdot 001)$ enhanced when a plasmid expression ChREBP was cotransfected with a TXNIP reporter vector into HepG2 cells (Fig. 3B). However, transient overexpression of c-Myc did not significantly $(P=0 \cdot 382)$ induce TXNIP promoter activity in the low glucose (1 mM) condition (Fig. 3C).

The effects of glucose are apparently mediated via a region located -420 to $-1 \mathrm{bp}$ upstream of the translational starting point of the TXNIP gene according to $5^{\prime}$-deletion reporter assays (Fig. 4A). Two putative ChoRE sequences, -430 to -414 GAGCACACCGTGTCCAC and -341 to -324 CACGAGGGCAGCACGAG (putative E-boxes underlined), were observed within this region. Two mutant reporter vectors, pChoREm1 and pChoREm2, were used to determine the specific ChoRE on the TXNIP gene. Following mutation of the putative -341 to -324 ChoRE from CACGAGGGCAGCACGAG to TTT GAGGGCAGCACGAG transient gene expression assays revealed that the reporter vector pChoREm2, but not pChoREm1, with the ChoRE mutation blocked the effect of $15 \mathrm{mM}$ glucose treatment (Fig. 4B and C). These results indicate that the effects of glucose are mediated by a region located -341 to $-324 \mathrm{bp}$ upstream of the translational starting point of the TXNIP gene. Transient gene expression assays revealed that the promoter activity of TXNIP gene was upregulated by treatment with glucose or maltose but not with mannitol. The promoter activity of TXNIP was upregulated fourfold when LNCaP cells were treated with high concentrations $(30 \mathrm{mM})$ of 3-O-methylglucose, although 3-O-methylglucose does not undergo phosphorylation by glucokinase (Fig. 5A). These results 

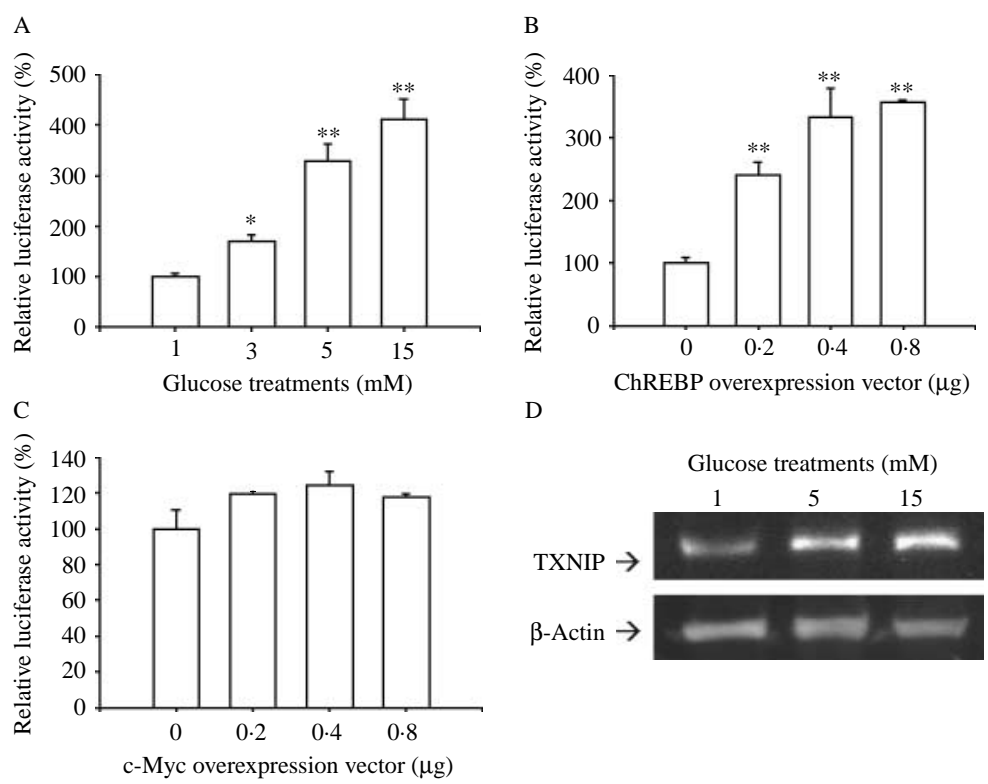

Figure 2 Effects of glucose on human TXNIP gene promoter activity in LNCaP cells. Reporter vector containing $5^{\prime}$-flanking region $(-1614$ to +1$)$ of TXNIP gene was constructed and transient gene reporter assay was performed as described in 'Materials and methods'. Experimental data are presented as mean ( \pm s.E.M.; $n=6)($ A) luciferase activity of reporter vectors. Regulation of TXNIP gene promoter activity by different glucose concentrations as indicated in LNCaP cells. Promoter activity after transient (B) overexpression of ChREBP or (C) c-Myc in LNCaP cells. (D) Cells were treated with different concentrations of glucose (as indicated) for $24 \mathrm{~h}$, then harvested and lysed to extract protein for immunoblot assay. (*: $\left.P<0.05 ;{ }^{* *}: P<0 \cdot 01\right)$.

were also found in HepG2 cells. Both concentrations $(15$ and $30 \mathrm{mM})$ of 3 -O-methylglucose treatment significantly induced TXNIP promoter activity in comparison with the $1 \mathrm{mM} 3-O$-methylglucose-treated group (Fig. 5B). Results of immunoblot assays also revealed similar findings (Fig. 5C). The effects of 3-Omethylglucose may be mediated via the same putative ChoRE with glucose treatment since mutation of the ChoRE from GAGCACACCGTGTCCAC $(-430$ to -414) to ACACCGTCCCCACGCG did not abolish the activating effects of $30 \mathrm{mM} \mathrm{3-O-methylglucose}$ on TXNIP promoter activity. However, mutation of the ChoRE from CACGAGGGCAGCACGAG ( -341 to $-324)$ to TTTGAGGGCAGCACGAG blocked $50 \%$ of the activating effects of $30 \mathrm{mM} \mathrm{3-O-methylglucose}$ on TXNIP promoter activity in LNCaP cells (Fig. 5D). Similar results were also found using the HepG2 cells (data not shown). The glucose-upregulated promoter activity of the TXNIP gene was not abolished when cells were cotreated with $10 \mathrm{mM}$ of the cell-permeable antioxidant ( $N$-acetylcysteine) and glucose (Fig. 5E). These findings suggest that TXNIP is transcriptioninduced by increased metabolism-dependent or -independent responses, but not by increased oxidants.

Electrophoretic mobility shift assays indicated the specific binding characteristics of the ChoRE in the promoter region of the human TXNIP gene. The nuclear extract from LNCaP cells treated with varying glucose concentration ( 1 and $15 \mathrm{mM}$ ) specifically bonded to the $\mathrm{P}^{32}$ labeled double-stranded oligonucleotide containing the putative ChoRE in different intensities. The observed mobility shift intensity was decreased significantly when the reaction mixture was challenged with unlabeled double-stranded oligonucleotides containing the ChoRE (Fig. 6).

\section{Discussion}

TXNIP mediates oxidative stress via suppression of TRX, a thio reductase with many cellular functions (Junn et al. 2000, Nishiyama et al. 2001). TXNIP is involved in several biological cellular events, especially cellular viability, through interaction with TRX (Schulze et al. 2002, Wang et al. 2002, Yoshida et al. 2005). A clinical study has indicated that glucose intolerance is associated with the high TRX levels in the plasma of diabetes mellitus patients (Miyamoto et al. 2005). Moreover, several in vivo and in vitro studies have revealed that deregulated TRX causes abnormal nutrition signals, which indicates that TXNIP is involved in the integrated regulation of glucose and 


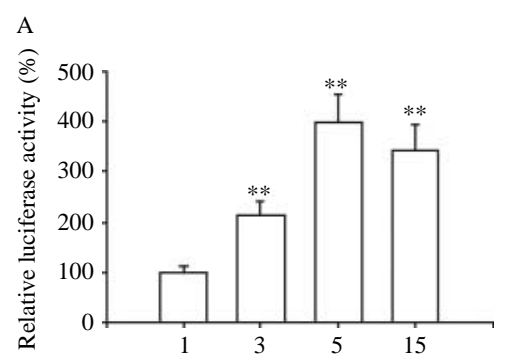

Glucose treatments $(\mathrm{mM})$

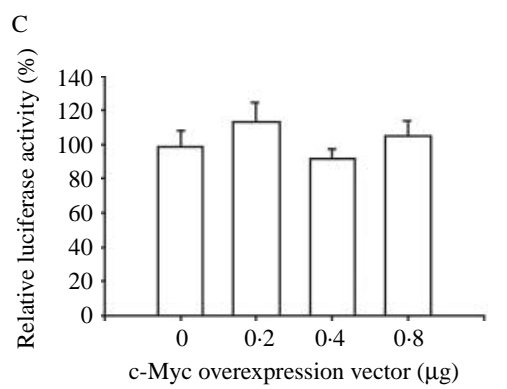

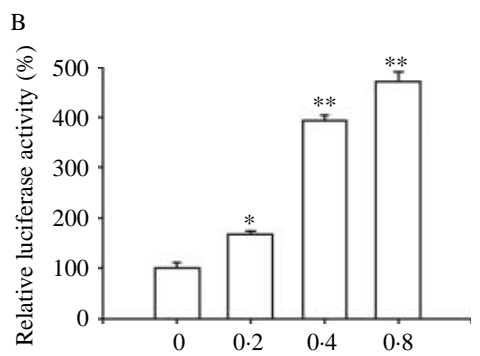

ChREBP overexpression vector $(\mu \mathrm{g})$

D

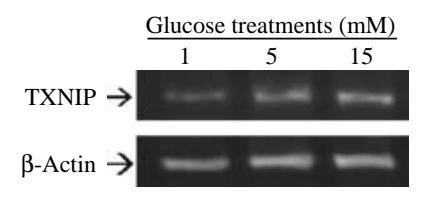

Figure 3 Effects of glucose on human TXNIP gene promoter activity in HepG2 cells. Reporter vector containing $5^{\prime}$-flanking region $(-1614$ to +1$)$ of TXNIP gene was constructed and transient gene reporter assay was performed as described in Fig. 2. (A) Regulation of TXNIP gene promoter activity by different concentrations of glucose as indicated in HepG2 cells. Promoter activity after transient (B) overexpression of ChREBP or (C) c-Myc in HepG2 cells. (D) Cells were treated with different concentrations of glucose (as indicated) for $24 \mathrm{~h}$. Cells were harvested and lysed to extract protein for immunoblot assay. $\left({ }^{\star}: P<0.05 ;{ }^{\star *}: P<0.01\right)$.
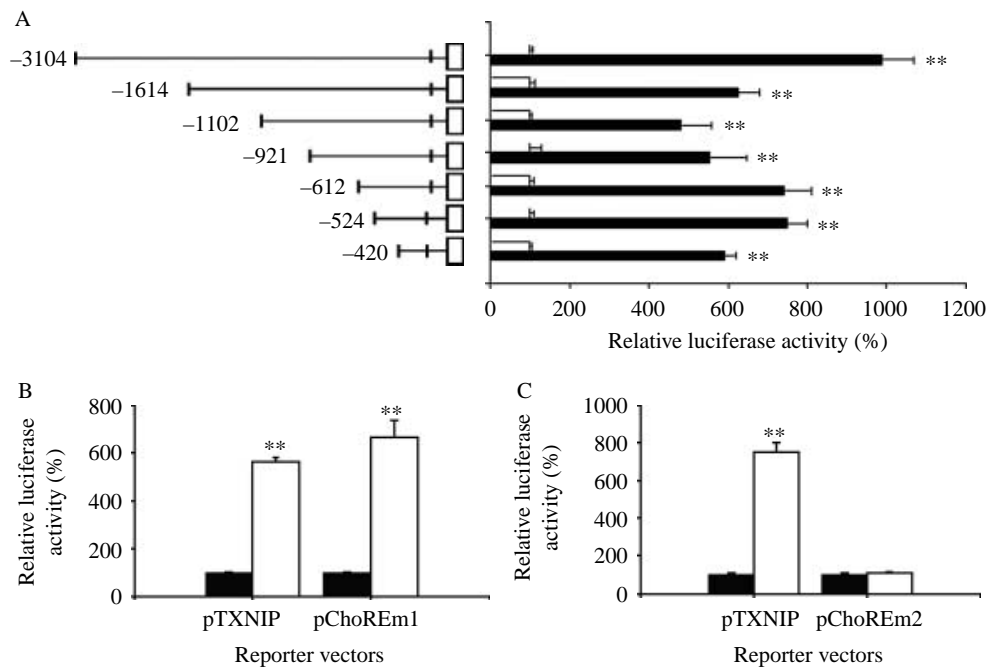

Figure 4 Glucose treatment modulation of human TXNIP gene is dependent on the putative carbohydrate response element. (A) $5^{\prime}$-deletion assay shows that glucose regulation of TXNIP gene promoter activity depends on the $(-524$ to +1$)$ DNA fragment. (B) The TXNIP promoter-luciferase reporter pTXNIP $(-524$ to +1 ) vector or mutant TXNIP promoter-luciferase reporter pChoREm1 or (C) pChoREm2 was transfected into LNCaP cells and transfected cells were treated with $1 \mathrm{mM}$ glucose (black bars) or $15 \mathrm{mM}$ glucose (white bars) for $16 \mathrm{~h}$.

Experimental data are presented as mean percentage ( \pm S.E.M.; $n=6$ ) luciferase activity produced by $15 \mathrm{mM}$ glucose treatment relative to $1 \mathrm{mM}$ glucose-treated samples. ( $\left.{ }^{\star \star}: P<0 \cdot 01\right)$. 

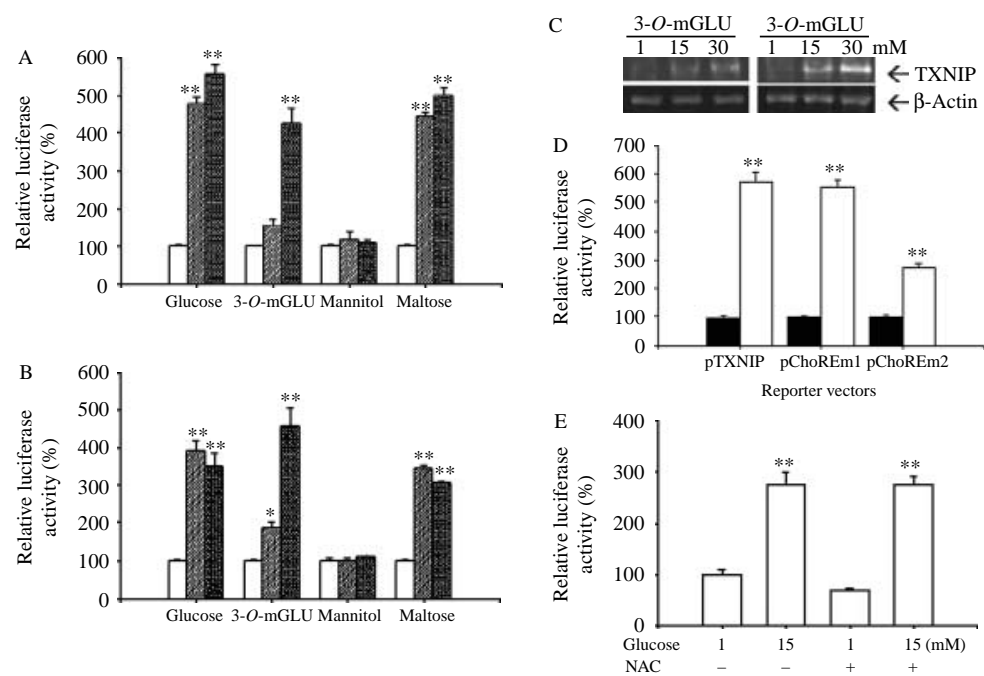

Figure 5 Modulation by glucose, 3-O-methylglucose, and antioxidants of TXNIP promoter activity. TXNIP $(-524$ to +1$)$ reporter vector-transfected $(A)$ LNCaP cells and (B) HepG2 cells were treated with different concentrations (white boxes, $1 \mathrm{mM}$; gray boxes, $15 \mathrm{mM}$; black boxes, $30 \mathrm{mM}$ ) of glucose, maltose, 3-Omethylglucose (3-O-mGLU), and mannitol for $24 \mathrm{~h}$. (C) LNCaP cells (left) and HepG2 cells (right) were treated with different concentrations of 3-Omethylglucose (as indicated) for $24 \mathrm{~h}$. Cells were harvested and lysed to extract protein for immunoblot assay. (D) The TXNIP reporter vectors-transfected LNCaP cells, as indicated, were treated with $1 \mathrm{mM} \mathrm{3-O-methylglucose} \mathrm{(black}$ bars) or $30 \mathrm{mM} 3-0$-methylglucose (white bars) for $24 \mathrm{~h}$. Experimental data are presented as mean percentage ( \pm S.E.M.; $n=6$ ) of luciferase activity produced by $30 \mathrm{mM} 3-\mathrm{O}$-methylglucose treatment relative to $1 \mathrm{mM} 3-O$-methylglucosetreated samples. (E) The TXNIP reporter vector-transfected LNCaP cells were treated with 1 or $15 \mathrm{mM}$ of glucose as indicated with or without $10 \mathrm{mM}$ of $N$ acetylcysteine (NAC) for $24 \mathrm{~h}$. Experimental data are presented as mean percentage \pm S.E.M. $(n=6)$ of luciferase activity induced by different treatments relative to the $1 \mathrm{mM}$ glucose treatment group. $\left({ }^{\star}: P<0.05{ }^{\star *}: P<0.01\right)$.

lipid metabolism (Hui et al. 2004, Schulze et al. 2004, Sheth et al. 2005, Oka et al. 2006). In two recent studies, oligonucleotide microarray analysis of intact human pancreatic islets and rat fibroblasts has indicated that TXNIP is upregulated by glucose (Hirota et al. 2002, Shalev et al. 2002). These studies suggest that TXNIP participates in the regulation of glucose metabolism, although the precise mechanisms are still unknown.

Increased glucose consumption is a common characteristic of malignant cells and is linked to increased energy production from aerobic glycolysis. Malignant cells exhibit altered metabolic patterns with increased reliance on anaerobic metabolism of glucose to lactic acid, even in the presence of abundant oxygen (Hochachka et al. 2002). Expressions of glucose transporter proteins 1 and 12 have been demonstrated in prostate cancer cells and tissues (Chandler et al. 2003). Other studies have also indicated that glucose uptake is increased in prostate cancer cells and that high rates of glucose consumption are required for rapid proliferation of androgen-independent prostate cancer cells (Singh et al. 1999). However, events involving the regulatory mechanisms of glucose on gene profiles of the human prostate are still not well known. In the present study, glucose-upregulated TXNIP gene expression was observed both in the prostate and hepatocellular carcinoma cells (HepG2) suggesting that modulation of glucose at the TXNIP gene may persist in both metabolic and non-metabolic organs.

Metabolites clearly regulate gene transcription via metabolic pathways for carbohydrates, fatty acids, and triglycerides (Towle 1995). Results from several independent laboratories have demonstrated that glucose may induce gene expression in the glycolytic pathway as well as coordinate long-term control of the enzymes required for fatty acid and triglyceride synthesis (Vaulont et al. 2000, Veech 2003). Moreover, the effects of glucose regulation on gene expression are dependent on the ChoRE within the promoter of the responsible gene which is recognized by ChREBP (Rufo et al. 2001, Yamashita et al. 2001, Elsas et al. 2002). When the ChREBP gene is knocked-out, rat hepatocytes exhibit diminished glucose regulation of lipogenic enzymes (Ishii et al. 2004). Overexpressed 


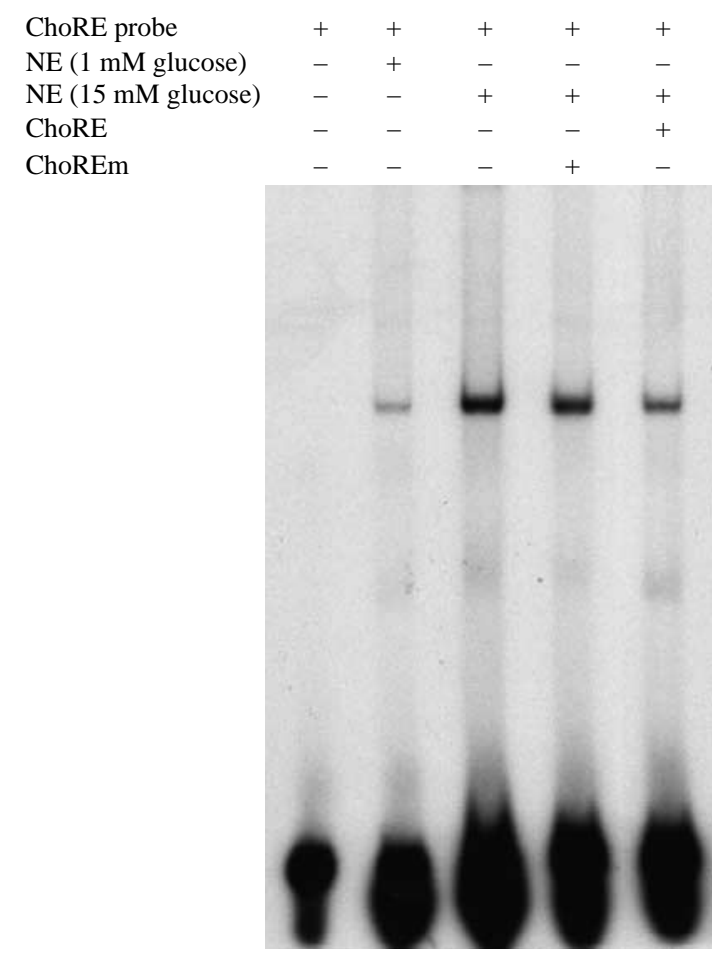

Figure 6 Glucose treatment induces nuclear proteins of LNCaP cell binding to the putative carbohydrate response element of TXNIP promoter. Electrophoretic mobility shift assay was performed as described in 'Materials and methods' using the $25 \mathrm{bp}$ ChoRE oligonucleotide probe end-labeled with ${ }^{32} \mathrm{P}$ (ChoRE probe) and nuclear extract from LNCaP cells (NE) after treatment with different concentrations of glucose as indicated. Gel shift intensity decreased when the reaction mixture was challenged with unlabeled double-stranded oligonucleotide (ChoRE) containing the carbohydrate response element but not when challenged with oligonucleotide featuring a mutation at the ChREBP binding site (ChoREm).

ChREBP in primary hepatocytes induces ChoREcontaining L-type pyruvate kinase and acetyl-CoA carboxylase $\alpha$ activity (Yamashita et al. 2001, Ma et al. 2005). The consensus sequence of ChoRE is composed of two palindromic E-box (CACGTG) or E-box-like sequences separated by five nucleotides (Rufo et al. 2001, Yamashita et al. 2001). However, other studies have suggested that the E-boxes of ChoRE are separated by varying numbers of nucleotides (Portois et al. 1999, Elsas et al. 2002, Wang \& Wollheim 2002). In this study, transient gene expression with $5^{\prime}$-deletion assay indicates that the effect of glucose modulation on the promoter activity of TXNIP depends on the DNA fragment located -420 to $-1 \mathrm{bp}$ upstream of the translational starting point of the TXNIP gene. Although two putative ChoRE sequences, -430 to -414 and -341 to -324 , were observed within this region ( -524 to -1$)$, transient gene expression assays revealed that only the ChoRE mutation from CACGAGGGCAGCACGAG to TTTGAGGGCAGCACGAG blocked the effects of $15 \mathrm{mM}$ glucose treatment. The putative ChoRE also was confirmed by EMSA. Although a previous report (Minn et al. 2005) predicted the same putative ChoRE in the human TXNIP gene using in silico sequence analysis, the current study clearly demonstrates that the effects of glucose are mediated via a region located -341 to $-324 \mathrm{bp}$ upstream of the translational starting point of the TXNIP gene (Fig. 4B and $\mathrm{C}$ ).

The E-box is also recognized by other transcription factors such as c-Myc, which is known to be required for glucose-mediated induction of metabolic enzyme genes (Osthus et al. 2000, Collier et al. 2003). The current study demonstrates that overexpression of ChREBP in prostate carcinoma cells induces activity at the ChoRE-containing TXNIP promoter in low glucose conditions. However, experimental results reveal no evidence of c-Myc participation in gene regulation of glucose on TXNIP. The TXNIP reporter vector, containing a deletion of the region -3104 to $-1614 \mathrm{bp}$ upstream of the translational starting point, demonstrated only $40 \%$ of the inductive response to glucose, compared with the full promoter. However, there was no consensus sequence of ChoRE found in this region $(-3104$ to -1614$)$. Therefore, results suggest that other unidentified transcription factors may be involved in the modulation of glucose induction of TXNIP promoter activity.

In the present study, we demonstrate that high dose (30 mM) 3-O-methylglucose can increase TXNIP promoter activity in both LNCaP and HepG2 cells, even without the 3-O-methylglucose phosphorylation. Moreover, it seems that HepG2 cells are more sensitive to 3-O-methylglucose treatments than LNCaP cells; $15 \mathrm{mM} 3-\mathrm{O}$-methylglucose treatment induced a twofold increase in TXNIP promoter activity in HepG2 cells. These results are similar to previous studies, which have indicated that 3-O-methylglucose induces TXNIP expression through glucose metabolism-independent pathways in human pancreatic $\beta$-cells (INS-1; Minn et al. 2005, 2006). Glucose and 3-O-methylglucose activation of TXNIP gene expression might be dependent on the same putative ChoRE. However, mutant ChoRE (-341 to -324) from CACGAGGGCAGCACGAG to TTTGAGGGCAGCACGAG only abolished $50 \%$ of the activating effects of $30 \mathrm{mM} 3-O$-methylglucose on TXNIP promoter activity (Fig. 5D). Our experiments, using mannitol to exclude an osmotic effect, suggest that unidentified factors or signals, other than ChREBP, are required for modulation of TXNIP promoter activity by $3-O$-methylglucose.

Although TXNIP modulates cell proliferation by interacting with TRX, the extent to which the effect of deregulated glucose on TXNIP gene expression is dependent on the TRX in the prostate is yet unknown. Previous studies have found suppression of TXNIP expression by hydrogen peroxide is inhibited by a 
cell-permeable antioxidant, $\mathrm{N}$-acetylcysteine (Schulze et al. 2002, Wang et al. 2002). In the present study, transient gene expression assay revealed that upregulation of TXNIP gene expression by glucose was not inhibited by $\mathrm{N}$-acetylcysteine suggesting that TRX may not play a pivotal role in this modulation of glucose.

This study demonstrates the expression of TXNIP in prostate carcinoma cells and the high metastasis rate of cells with lower TXNIP abound than normal or low metastasis prostate cells. This study is the first report that demonstrates the effects of upregulated glucose on TXNIP gene expression in both prostate carcinoma cells and hepatocellular carcinoma cells. Glucose modulates TXNIP gene expression via a ChoRE in the TXNIP gene promoter through the ChREBP pathway, but not the c-Myc or TRX pathway.

\section{Declaration of interest}

The authors declare that there is no conflict of interest that would prejudice the impartiality of the research reported.

\section{Funding}

This Research was supported by Chang Gung Memorial Hospital (CMRPD170471), Terry Fox Cancer Foundation (SMRPG340041), and Taiwan National Science Council (93-2314-B-182A-092 and 972320-B-182-023-MY3).

\section{References}

Aitken CJ, Hodge JM, Nishinaka Y, Vaughan T, Yodoi J, Day CJ, Morrison NA \& Nicholson GC 2004 Regulation of human osteoclast differentiation by thioredoxin binding protein-2 and redox-sensitive signaling. Journal of Bone and Mineral Research 19 2057-2064.

Chandler JD, Eillians ED, Slavin JL, Best JD \& Rogers S 2003 Expression and localization of GLUT1 and GLUT12 in prostate carcinoma. Cancer 97 2035-2042.

Chen KS \& DeLuca HF 1994 Isolation and characterization of a novel cDNA from HL-60 cells treated with 1,25-dihydroxyvitamin D-3. Biochimica et Biophysica Acta 1219 26-32.

Collier JJ, Doan TT, Daniels CJ, Schurr R, Kolls JK \& Scott DK 2003 $\mathrm{c}-\mathrm{Myc}$ is required for the glucose-mediated induction of metabolic enzyme genes. Journal of Biological Chemistry 278 6588-6595.

Dutta KK, Nishinaka Y, Masutani H, Akatsuka S, Aung TT, Shirase T, Lee WH, Yamada Y, Hiai H, Yodoi J et al. 2005 Two distinct mechanisms for loss of thioredoxin-binding protein-2 in oxidative stress-induced renal carcinogenesis. Laboratory Investigation 85 798-807.

Elsas LJ, Webb AL \& Langley SD 2002 Characterization of a carbohydrate response element regulating the gene for human galactose-1-phosphate uridyltransferase. Molecular Genetics and Metabolism 76 287-296.

Feng TH, Tsui KH \& Juang HH 2005 Cholesterol modulation of the expression of mitochondrial aconitase in human prostatic carcinoma cells. Chinese Journal of Physiology 48 93-100.
Filby CE, Hooper SB, Sozo F, Zahra VA, Flecknoe SJ \& Wallace MJ 2006 VDUP1: a potential mediator of expansion-induced lung growth and epithelial cell differentiation in the ovine fetus. American Journal of Physiology 290 L250-L258.

Goldberg SF, Miele ME, Hatta N, Takata M, Paquette-Straub C, Freedman LP \& Welch DR 2003 Melanoma metastasis suppression by chromosome 6: evidence for a pathway regulated by CRSP3 and TXNIP. Cancer Research 63 432-440.

Gulati P, Klohn PC, Krug H, Gottlicher M, Markova B, Bohmer FD \& Herrlich P 2001 Redox regulation in mammalian signal transduction. IUBMB Life $\mathbf{5 2} 25-28$.

Han SH, Jeon JH, Ju HR, Jung U, Kim KY, Yoo HS, Lee YH, Song KS, Hwang HM, Na YS et al. 2003 VDUP1 upregulated by TGF-betal and 1,25-dihydroxyvitamin D3 inhibits tumor cell growth by blocking cell-cycle progression. Oncogene 22 4035-4046.

Hirota T, Okano T, Kokame K, Shirotani-Ikejima H, Miyata T \& Fukada Y 2002 Glucose down-regulates Per1 and Per2 mRNA levels and induces circadian gene expression in cultured Rat-1 fibroblasts. Journal of Biological Chemistry 277 44244-44251.

Hochachka PW, Rupert JL, Goldenberg L, Gleave M \& Kozlowski P 2002 Going malignant: the hypoxia - cancer connection in the prostate. BioEssays 24 749-757.

Hui TY, Sheth SS, Diffey JM, Potter DW, Lusis AJ, Attie AD \& Davis RA 2004 Mice lacking thioredoxin-interacting protein provide evidence linking cellular redox state to appropriate response to nutritional signals. Journal of Biological Chemistry $\mathbf{2 7 9}$ 24387-24393

Ikarashi M, Takahashi Y, Ishii Y, Nagata T, Asai S \& Ishikawa K 2002 Vitamin D3 up-regulated protein 1 (VDUP1) expression in gastrointestinal cancer and its relation to stage of disease. Anticancer Research 22 4045-4048.

Ishii S, IIzuka K, Miller BC \& Uyeda K 2004 Carbohydrate response element binding protein directly promotes lipogenic enzyme gene transcription. PNAS 101 15597-15602.

Jeon JH, Lee KN, Hwang CY, Kwon KS, You KH \& Choi I 2005 Tumor suppressor VDUP1 increases p27(kip1) stability by inhibiting JAB1. Cancer Research 65 4485-4489.

Juang HH, Hsieh ML \& Tsui KH 2004 Testosterone modulates mitochondrial aconitase in the full-length human androgen receptor-transfected PC-3 prostatic carcinoma cells. Journal of Molecular Endocrinology 33 121-132.

Junn E, Han SH, Im JY, Yang Y, Cho EW, Um HD, Kim DK, Lee KW, Han PL, Rhee SG et al. 2000 Vitamin D3 up-regulated protein 1 mediates oxidative stress via suppressing the thioredoxin function. Journal of Immunology 164 6287-6295.

Kobayashi T, Uehara S, Ikeda T, Itadani H \& Kotani H 2003 Vitamin D3 up-regulated protein-1 regulates collagen expression in mesangial cells. Kidney International 64 1632-1642.

Lee KN, Kang HS, Jeon JH, Kim EM, Yoon SR, Song H, Lyu CY, Piao ZH, Kim SU, Han YH et al. 2005 VDUP1 is required for the development of natural killer cells. Immunity 2 195-208.

Ma L, Tsatsos NG \& Towle HC 2005 Direct role of ChREBP-Mlx in regulating hepatic glucose responsive genes. Journal of Biological Chemistry 280 12019-12027.

Minn AH, Hafele C \& Shalev A 2005 Thioredoxin-interating protein is stimulated by glucose through a carbohydrate response element and induces $\beta$-cell apoptosis. Endocrinology 146 2397-2405.

Minn AH, Couto FM \& Shalev A 2006 Metabolism-independent sugar effects on gene transcription: the role of 3-O-methylglucose. Biochemistry 45 11047-11051.

Miyamoto S, Kawano H, Hokamaki J, Soejima H, Kojima S, Kudoh T, Nagayoshi Y, Sugiyama S, Sakamoto T, Yoshimura M et al. 2005 Increased plasma levels of thioredoxin in patients with glucose intolerance. Internal Medicine 44 1127-1132.

Nishinaka Y, Nishiyama A, Masutani H, Oka S, Ahsan KM, Nakayama Y, Ishii Y, Nakamura H, Maeda M \& Yodoi J 2004 Loss of thioredoxinbinding protein-2/vitamin D3 up-regulated protein 1 in human 
cell leukemia virus type I-dependent T-cell transformation: implications for adult T-cell leukemia leukemogenesis. Cancer Research 64 1287-1292.

Nishiyama A, Masutani H, Nakamura H, Nishinaka Y \& Yodoi J 2001 Redox regulation by thioredoxin and thioredoxin-binding proteins. IUBMB Life 52 29-33.

Oka S, Liu W, Masutani H, Hirata H, Shinkai Y, Yamada S, Yoshida T, Nakamura H \& Yodoi J 2006 Impaired fatty acid utilization in thioredoxin binding protein-2 (TBP-2)-deficient mice: a unique animal model of Reye syndrome. FASEB Journal 20 121-123.

Osthus RC, Shim H, Kim S, Li Q, Reddy R, Mukherjee M, Xu Y, Wonsey D, Lee LA \& Dang CV 2000 Deregulation of glucose transporter 1 and glycolytic gene expression by c-Myc. Journal of Biological Chemistry 274 21797-21800.

Pang ST, Dillner K, Wu X, Pousette A, Norstedt G \& Flores-Morales A 2002 Gene expression profiling of androgen deficiency predicts a pathway of prostate apoptosis that involves genes related to oxidative stress. Endocrinology 143 4897-4906.

Parikh H, Carlsson E, Chutkow WA, Johansson LE, Storgaard H, Poulsen P, Saxena R, Ladd C, Schulze PC, Mazzini MJ et al. 2007 TXNIP regulates peripheral glucose metabolism in humans. PLoS Medicine 4 e158.

Portois L, Maget B, Tastenoy M, Perret J \& Svobda M 1999 Identification of a glucose response element in the promoter of the rat glucagon receptor gene. Journal of Biological Chemistry 274 $8181-8190$

Rufo C, Teran-Garcia M, Nakamura MT, Koo S-H, Towle HC \& Clarke SD 2001 Involvement of a unique carbohydrate-responsive factor in the glucose regulation of rat liver fatty-acid synthase gene transcription. Journal of Biological Chemistry 276 21969-21975.

Schulze PC, De Keulenaer GW, Yoshioka J, Kassik KA \& Lee RT 2002 Vitamin D3 up-regulated protein-1 (VDUP1) regulates redoxdependent vascular smooth muscle cell proliferation through interaction with thioredoxin. Circulation Research 91 689-695.

Schulze PC, Yoshioka J, Takahashi T, He Z, King GL \& Lee RT 2004 Hyperglycemia promotes oxidative stress through inhibition of thioredoxin function by thioredoxin-interacting protein. Journal of Biological Chemistry 279 30369-30374.

Shalev A, Pise-Masison CA, Radonovich M, Hoffmann SC, Hirshberg B, Brady JN \& Harlan DM 2002 Oligonucleotide microarray analysis of intact human pancreatic islets: identification of glucose-responsive genes and a highly regulated TGF $\beta$ signaling pathway. Endocrinology 143 3695-3698.

Sheth SS, Castellani LW, Chari S, Wagg C, Thipphavong CK, Bodnar JS, Tontonoz P, Attie AD, Lopaschuk GD \& Lusis AJ 2005 Thioredoxininteracting protein deficiency disrupts the fasting-feeding metabolic transition. Journal of Lipid Research 46 123-134.

Simmons DG \& Kennedy TG 2004 Rat endometrial Vdup1 expression: changes related to sensitization for the decidual cell reaction and hormonal control. Reproduction 127 475-482.

Singh G, Lakkis CL, Laucirica R \& Epner DE 1999 Regulation of prostate cancer cell division by glucose. Journal of Cellular Physiology $180309-314$.

Song H, Cho D, Jeon JH, Han SH, Hur DY, Kim YS \& Choi I 2003 Vitamin D3 up-regulating protein 1 (VDUP1) antisense DNA regulates tumorigenicity and melanogenesis of murine melanoma cells via regulating the expression of Fas ligand and reactive oxygen species. Immunology Letters 86 235-247.
Towle HC 1995 Metabolic regulation of gene transcription in mammals. Journal of Biological Chemistry 27023235 -23238.

Tsui KH, Chang PL \& Juang HH 2006 Zinc blocks gene expression of mitochondrial aconitase in human prostatic carcinoma cells. International Journal of Cancer 118 609-615.

Tsui KH, Hsieh WC, Lin MH, Chang PL \& Juang HH 2008 Triiodothyronine modulates cell proliferation of human prostatic carcinoma cells by downregulation of the B-cell translocation gene 2 . Prostate 68 610-619.

Vaulont S, Vasseur-Cognet M \& Kahn A 2000 Glucose regulation of gene transcription. Journal of Biological Chemistry 27531555 - 31558.

Veech RL 2003 A humble hexose monophosphate pathway metabolite regulates short- and long-term control of lipogenesis. PNAS 100 $5578-5580$

Wang H \& Wollheim CB 2002 ChREBP rather than USF2 regulates glucose stimulation of endogenous L-pyruvate kinase expression in insulin-secreting cells. Journal of Biological Chemistry 277 32746-32752.

Wang Y, De Keulenaer GW \& Lee RT 2002 Vitamin D3-up-regulated protein-1 is a stress-responsive gene that regulates cardiomyocyte viability through interaction with thioredoxin. Journal of Biological Chemistry 277 26496-26500.

Wang Z, Rong YP, Malone MH, Davis MC, Zhong F \& Distelhorst CW 2006 Thioredoxin-interacting protein (txnip) is a glucocorticoidregulated primary response gene involved in mediating glucocorticoid-induced apoptosis. Oncogene 25 1903-1913.

Weijerman PC, Konig JJ, Wong ST, Niesters HG \& Peehl DN 1994 Lipofection-mediated immortalization of human prostatic epithelial cells of normal and malignant origin using human papillomavirus type 18 DNA. Cancer Research 54 5579-5583.

Xiang G, Seki T, Schuster MD, Witkowski P, Boyle AJ, See F, Martens TP, Kocher A, Sondermeijer H, Krum H et al. 2005 Catalytic degradation of vitamin D up-regulated protein 1 mRNA enhances cardiomyocyte survival and prevents left ventricular remodeling after myocardial ischemia. Journal of Biological Chemistry 280 39394-39402.

Yamashita H, Takenoshita M, Sakurai M, Bruick RK, Henzel WJ, Shillinglaw W, Armot D \& Uyeda K 2001 A glucose-responsive transcription factor that regulates carbohydrate metabolism in the liver. PNAS 98 9116-9121.

Yamawaki H, Pan S, Lee RT \& Berk BC 2005 Fluid shear stress inhibits vascular inflammation by decreasing thioredoxin-interacting protein in endothelial cells. Journal of Clinical Investigation 115 $733-738$.

Yoshida T, Nakamura H, Masutani H \& Yodoi J 2005 The involvement of thioredoxin and thioredoxin binding protein-2 on cellular proliferation and aging process. Annals of the New York Academy of Sciences 1055 1-12.

Yoshioka J, Schulze PC, Cupesi M, Sylvan JD, MacGillivray C, Gannon J, Huang H \& Lee RT 2004 Thioredoxin-interacting protein controls cardiac hypertrophy through regulation of thioredoxin activity. Circulation 109 2581-2586.

Received in final form 20 November 2008

Accepted 2 December 2008

Made available online as an Accepted Preprint 3 December 2008 